\title{
ESTEATOSE E ESTEATOHEPATITE NÃO-ALCOÓLICA NOS PACIENTES COM HEPATITE CRÔNICA PELO VÍRUS DA HEPATITE C
}

\author{
Gabriela CORAL, Angelo Alves de MATTOS, Ângelo Zambam de MATTOS e \\ Diogo Edele dos SANTOS
}

RESUMO - Racional - Esteatose hepática é achado comum na hepatite pelo vírus C (VHC), principalmente nos pacientes com genótipo 3, podendo estar relacionada à resposta ao tratamento antiviral e prognóstico da hepatite crônica. Objetivo - Determinar a presença de esteatose e de esteatohepatite não-alcoólica na hepatite crônica pelo VHC, correlacionando-as com o genótipo do VHC e com o grau de fibrose hepática. Pacientes e métodos - Foram avaliados retrospectivamente 120 pacientes com hepatite crônica pelo VHC. A genotipagem foi avaliada em 102 pacientes. Todos os espécimes de biopsia hepática foram submetidos as colorações: picrosirius, hematoxilina-eosina e perls. O estádio da hepatite $\mathrm{C}$ foi realizado de acordo com a Classificação Brasileira e o diagnóstico de esteatohepatite nãoalcoólica estabelecido de acordo com os critérios da American Association for the Study of Liver Diseases. O nível de significância considerado na analise estatística foi de 5\%. Resultados - A esteatose foi encontrada em 65 dos 120 pacientes $(54,2 \%)$, sendo leve em 37/65 (56,9\%), moderada em 12/65 (18,5\%) e intensa em 10/65 (15,4\%). Quanto à fibrose, 80 de 120 pacientes (66,6\%) tinham fibrose entre F0 e F2, e 40 (33,3\%) tinham fibrose F3 ou F4. Esteatose foi mais freqüente no genótipo $3(76,7 \%)$ em relação aos demais genótipos $(49,0 \%)$. Não houve diferença na freqüência de esteatose quando comparados os pacientes com F3/F4 (52,5\%) e demais graus de fibrose $(54,4 \%)$. Esteatohepatite não-alcoólica foi diagnosticada em 8/120 casos (6,7\%), sendo significativamente correlacionada com o genótipo 3 e com fibrose avançada (F3/F4). Conclus̃̃es - A presença de esteatose, assim como de esteatohepatite não-alcoólica na hepatite $\mathrm{C}$, estão relacionados ao genótipo 3 , sendo a esteatohepatite não-alcoólica correlacionada a graus mais avançados de fibrose.

DESCRITORES - Fígado gorduroso. Hepatite C crônica.

\section{INTRODUÇÃO}

Esteatose hepática, agregado e/ou folículo linfóide e lesão do epitélio do ducto biliar são achados histológicos que sugerem infecção pelo vírus da hepatite $\mathrm{C}$ $(\mathrm{VHC})^{(4,27)}$. Por outro lado, a esteatose hepática também pode estar presente em outras condições, associadas ou não com a infecção pelo VHC, como ingestão de álcool, diabete melito (DM), obesidade e medicamentos de uso comum (por exemplo, anti-inflamatórios não-esteróides e corticoesteróides $)^{(8,9,11,30)}$.

A doença gordurosa não-alcoólica do fígado (DGNA), abrangendo o espectro de esteatose - a esteatohepatite, é um diagnóstico freqüente nos pacientes com obesidade, $\mathrm{DM} \mathrm{e}$ síndrome metabólica ${ }^{(29)} \mathrm{e}$, recentemente, tem sido diagnosticada em associação com a hepatite pelo $\mathrm{VHC}^{(26,32)}$.

A esteatose, como resultado da infecção pelo VHC, parece ser causada pelo vírus de genótipo 3 Há evidência de que a esteatose, na infecção por vírus deste genótipo, resulte da ação direta do VHC, ou seja, de um efeito citotóxico $^{(18,19,23,25)}$. Por outro lado, quando há esteatose em pacientes infectados pelo genótipo 1 , esta freqüentemente encontra-se associada à síndrome metabólica e à resistência periférica à insulina ${ }^{(9,10)}$.

O objetivo deste estudo foi determinar a presença de esteatose e de esteatohepatite não-alcoólica (EHNA) na hepatite crônica pelo VHC, correlacionando-as com o genótipo do VHC e com o grau de fibrose hepática.

Serviço de Gastroenterologia Clinica e Cirúrgica da Irmandade Santa Casa de Misericórdia de Porto Alegre, Porto Alegre, RS

Endereço para correspondência: Dra. Gabriela Coral - Rua Ten. Cel. Fabricio Pillar, 179/501 - Moinhos de Vento - 90450-040 - Porto Alegre, RS. E-mail: g.coral@terra.com.br. 


\section{MATERIAL E MÉTODOS}

Este é um estudo retrospectivo em que foram analisados seqüencialmente, na Irmandade Santa Casa de Misericórdia de Porto Alegre, Porto Alegre, RS, pacientes com infecção crônica pelo VHC confirmada pela PCR (polimerase chain reaction) e que realizaram biopsia hepática no período de janeiro de 2003 a junho de 2005.

Foram excluídos da análise os pacientes com consumo de álcool diário igual ou maior que $40 \mathrm{~g}$ para homens e $20 \mathrm{~g}$ para mulheres e aqueles com associação de outras etiologias tais como hepatite pelo vírus $B$, hepatite autoimune, hemocromatose, doença de Wilson, deficiência de alfa-1-antitripsina ou uso de medicamentos hepatotóxicos. Biopsias com representação histológica de menos de 7 espaços-porta foram excluídas.

Após exclusão de 15 pacientes, 120 com hepatite crônica pelo VHC foram avaliados.

A genotipagem do VHC foi realizada através de técnica de polimorfismo fragmentado de restrição (RFLP-PCR) ${ }^{(16)}$.

Foram também avaliados o índice de massa corpórea (IMC) e a presença de DM. O IMC foi calculado pela fórmula peso/altura ${ }^{2}$. O diagnóstico de DM era determinado na presença de glicemia de jejum maior ou igual a $126 \mathrm{mg} / \mathrm{dL}$ em pelo menos dois exames ${ }^{(2)}$.

Todos os espécimes de biopsia hepática foram submetidos as seguintes colorações: picrosirius, hematoxilina-eosina e perls.

A hepatite $\mathrm{C}$ foi classificada de acordo com a Classificação Brasileira $^{(12)}$ em que F0 = ausência de fibrose, F1 = expansão fibrosa portal, F2 = expansão fibrosa portal com septos, F3 = transformação nodular, F4 = cirrose. Para análise, os pacientes foram agrupados de acordo com o grau de fibrose em: fibrose leve, F0 a F2 ou fibrose intensa, F3 e F4.

A esteatose foi classificada como grau 1 quando acometia menos de $33 \%$ dos hepatócitos; grau 2, 33\%-66\% e grau 3, quando acometia mais de $66 \%$. Esta foi posteriormente classificada como macrogoticular ou mista ${ }^{(5)}$.

O diagnóstico de EHNA foi estabelecido de acordo com os critérios da American Association for the Study of Liver Diseases $^{(22)}$, sendo necessários a presença de esteatose, infiltrado misto e/ou balonização hepatocelular, e/ou fibrose pericelular em área centro-lobular.

A presença de outros marcadores etiológicos da hepatite pelo VHC tais como agregado ou folículo linfóide e lesão do epitélio do ducto biliar foram também avaliados, bem como a presença de siderose.

O estudo foi aprovado pelo Comitê de Ética em Pesquisa da instituição onde se desenvolveu o trabalho.

Foram utilizados os testes qui-quadrado ou teste exato de Fischer, sendo o nível de significância considerado para análise estatística de 0,05 .

\section{RESULTADOS}

A média de idade dos 120 pacientes analisados foi de 46,5 $\pm 9,82$ anos (25-70), sendo 79 pertencentes ao gênero feminino $(65,8 \%)$ e 41 ao masculino $(34,2 \%)$.

Os dados de peso, altura e presença ou não de DM foram recuperados em 90 pacientes. Destes, o IMC foi $\geq$ a 30 em 11/90 $(12,2 \%)$ e DM esteve presente em 14/90 (15,5\%) casos.
Em 102 pacientes foi possível obter o genótipo, sendo a freqüência do genótipo 1 encontrada em $38 / 102(37,2 \%)$, genótipo 2 em 6/102 (5,8\%) e genótipo 3 em 58/102 (56,8\%).

Esteatose, na análise histopatológica, foi encontrada em 65 dos 120 pacientes $(54,2 \%)$, sendo leve em $37 / 65(56,9 \%)$, moderada em 12/65 (18,5\%) e intensa em 10/65 (15,4\%). A esteatose foi exclusivamente macrogoticular em 60 casos $(92,3 \%)$ e mista em 5 casos $(7,7 \%)$, sendo mais freqüente no genótipo $3(76,7 \%) \mathrm{em}$ relação aos demais genótipos $(49,0 \%)(P=0,021)$. Da mesma forma, esteatose intensa (graus 2 e 3 ) foi mais freqüentemente encontrada nos pacientes com genótipo $3(48,5 \%)$ em comparação ao encontrado em pacientes com os demais genótipos $(23,1 \%)(P=0,045)$.

A prevalência de DM não foi diferente entre os pacientes com ou sem esteatose $(P=0,09)$. Da mesma forma, obesidade não foi fator de risco para a presença de esteatose $(P=0,3)$

Quanto à fibrose, 80 dos 120 pacientes $(66,6 \%)$ tinham fibrose entre F0 e F2 e $40(33,3 \%)$ tinham fibrose F3 ou F4.

Não houve diferença na freqüência de esteatose quando comparados os pacientes com F3/F4 (52,5\%) e demais graus de fibrose $(54,4 \%)(P=0,84)$.

Agregado linfóide foi encontrado em 27/120 (22,5\%) e lesão de ducto biliar em 12/120 (10\%). Siderose foi encontrada em $14 / 120(11,7 \%)$.

EHNA foi diagnosticada em $8 / 120$ casos $(6,7 \%)$, sendo significativamente correlacionada com o genótipo $3(P=0,030)$ e com fibrose avançada (F3/F4) $(P=0,029)$.

Não houve correlação entre EHNA e obesidade $(P=0,63)$ ou $\mathrm{DM}(P=0,78)$.

\section{DISCUSSÃO}

A esteatose está presente em $31 \%$ a $65 \%$ dos casos de hepatite crônica pelo $\mathrm{VHC}^{(3,8,15,17,24,25)}$. Na presente casuística, o achado de esteatose em $54 \%$ dos casos é próximo ao demonstrado na literatura ${ }^{(8,18)}$.

Em geral, a esteatose associada à hepatite crônica pelo VHC é leve $\mathrm{e}^{(3,6,25)}$, achado também demonstrado neste estudo, onde aproximadamente $60 \%$ dos casos tinham esteatose em menos de $33 \%$ dos hepatócitos.

Postula-se que a esteatose nos pacientes infectados pelo genótipo 3 esteja relacionada ao efeito citotóxico direto do VHC. MORIYA et al. ${ }^{(21)}$ demonstraram, em estudo experimental, que a proteína core do VHC induz esteatose em ratos transgênicos. A corroborar a ação deletéria do vírus, foi demonstrado que a esteatose nos pacientes com esse genótipo correlaciona-se com a carga viral ${ }^{(1,24)}$. Outra evidência a favor de efeito citotóxico direto do VHC genótipo 3 é o fato de que, após tratamento e eliminação do vírus, há diminuição significativa da esteatose ${ }^{(24)}$.

Interessante também é o estudo KUMAR et al..$^{(18)}$, que avaliaram 62 pacientes com hepatite crônica pelo VHC e esteatose antes e após o tratamento com interferon isolado ou com interferon associado à ribavirina. Nos pacientes com genótipo 1 , a despeito da resposta ao tratamento, não houve diferença no grau de esteatose entre a biopsia pré- e pós-tratamento. Por outro lado, nos pacientes com genótipo 3 e resposta viral sustentada, houve melhora significativa da esteatose, sendo que em $71 \%$ dos casos ela desapareceu e em $14 \%$, reduziu pelo menos 1 grau. 
CASTÉRA et al.(7) também demostraram melhora da esteatose após resposta viral sustentada, achado correlacionado exclusivamente ao genótipo 3. Nesse estudo, após análise multivariada, foi demonstrado que resposta viral sustentada, genótipo 3, esteatose intensa e IMC maior que $25 \mathrm{~kg} / \mathrm{m}^{2}$ estiveram associados à melhora da fibrose após tratamento.

A associação de esteatose com o genótipo viral, na hepatite crônica pelo VHC, também foi demonstrada na presente série, onde a esteatose foi encontrada em quase $80 \%$ dos pacientes com genótipo 3, ao contrário do ocorrido nos infectados pelos demais genótipos $(49,0 \%)$. Da mesma forma, esteatose intensa foi mais freqüentemente encontrada nos pacientes com esse genótipo.

Por outro lado, a esteatose no genótipo 1 tem sido associada com a presença da síndrome metabólica e resistência periférica à insulina. Fatores como idade, IMC e obesidade central têm sido relacionados ao desenvolvimento de esteatose associada a esse genótipo ${ }^{(9)}$.

$\mathrm{O}$ estudo de FARTOUX et al. ${ }^{(10)}$ demonstrou que o índice médio de HOMA (Homeostasis Model Assessment) para resistência insulínica estava significativamente aumentado nos pacientes com esteatose e genótipo 1, em comparação aos com esteatose e genótipo 3.

Da mesma forma, no estudo de ADINOLFI et al. ${ }^{(1)}$, a associação entre IMC e esteatose somente foi demonstrada no genótipo 1. Por outro lado, WESTIN et al. ${ }^{(30)}$ chamam a atenção que somente esteatose leve foi associada à obesidade, enquanto que esteatose intensa foi exclusivamente associada ao genótipo 3.

$\mathrm{Na}$ casuística em foco, não houve correlação entre a presença de esteatose e obesidade ou DM, achado este também já documentado na literatura ${ }^{(8)}$.

Com relação ao estádio histológico da hepatite pelo VHC, a maioria dos estudos demonstra associação entre esteatose e fibrose a partir de F2, ou seja, fibrose porta com $\operatorname{septos}^{(8,17,24)}$.

WESTIN et al ${ }^{(30)}$ chamam atenção ao fato de que a progressão da fibrose foi mais importante nos casos em que a primeira biopsia demonstrava esteatose, sendo esse efeito mais evidente nos pacientes com genótipo 3. Esses autores chegam a concluir pela necessidade de tratamento precoce para este grupo de pacientes (genótipo 3 e esteatose).

No estudo de WYATT et al. ${ }^{(31)}$, a presença de esteatose esteve correlacionada com a idade do paciente e com o estágio da fibrose. Nesse estudo, os autores comparam a presença de esteatose em biopsias seriadas e demonstraram que ela persiste e tem tendência a aumentar com o tempo.

CASTÉRA et al. ${ }^{(6)}$ também demonstraram piora da esteatose em 34\% em biopsia seqüencial, estabilização em $50 \%$ e melhora em $16 \%$. A piora de esteatose foi o único fator preditivo de piora da fibrose na segunda biopsia em uma análise multivariada.

Embora estudos documentem a relação entre esteatose e fases mais avançadas de fibrose, tem sido demonstrado que, na cirrose, em geral ocorre diminuição da esteatose ${ }^{(31)}$. Possíveis explicações para tal achado incluem: pobre estado nutricional dos pacientes com cirrose, alterações da circulação intra-hepática e shunt portossistêmico, ou ainda capilarização dos sinusóides ${ }^{(8)}$.

No presente estudo, não houve correlação entre esteatose e fibrose, no entanto, observou-se correlação entre presença de EHNA e fibrose avançada.

Histologicamente, a EHNA difere da hepatite crônica. Na primeira, o infiltrado em geral é lobular e misto, enquanto na hepatite crônica, o infiltrado é linfomononuclear, concentra-se preferencialmente no espaço porta e determina necrose periportal. A balonização traduz lesão hepatocelular, sugestiva de esteatohepatite. A fibrose da esteatohepatite é pericelular e perivenular em área centro-lobular enquanto que na hepatite $\mathrm{C}$, é portal. A esteatose deve necessariamente estar presente na esteatohepatite embora seja freqüente nas duas causas de hepatopatia ${ }^{(13,14)}$.

A prevalência de EHNA em associação com hepatite pelo VHC varia de $4 \%$ a $18 \%$ na literatura ${ }^{(20,32)}$. Na presente amostra foi de aproximadamente $7 \%$.

No estudo de YOUNOSSI et al. ${ }^{(32)}$ a obesidade, o genótipo 3 e a fibrose avançada foram associados com a presença de EHNA nos pacientes com hepatite crônica pelo VHC.

De forma semelhante, no estudo de SOLIS-HERRUZO et al. ${ }^{(28)}$, o IMC e a fibrose foram associados com EHNA na hepatite crônica pelo VHC.

Na presente casuística, a EHNA também foi associada ao genótipo 3 e à fibrose avançada, embora não tenha sido relacionada à obesidade ou à DM.

Conclui-se que a presença e a intensidade da esteatose, assim como o diagnóstico de EHNA na hepatite $\mathrm{C}$, estão relacionados ao genótipo 3, sendo a EHNA correlacionada a graus mais avançados de fibrose.

Coral G, Mattos AA, Mattos AZ, Santos DE. Steatosis and non-alcoholic steatohepatitis in patients with chronic hepatitis due to hepatitis C virus infection. Arq Gastroenterol. 2006;43(4):265-8.

ABSTRACT - Background - Hepatic steatosis is a common finding in patients with hepatitis $\mathrm{C}$, mainly virus $\mathrm{C}$ genotype 3 . Steatosis in these cases might be associated with antiviral treatment response and with prognosis of chronic hepatitis. Aims - To determine the presence of steatosis and non-alcoholic steatohepatitis in chronic hepatitis $\mathrm{C}$ and its correlation with genotype and hepatic fibrosis. Patients and methods - One hundred and twenty patients with chronic hepatitis $\mathrm{C}$ were retrospectively evaluated; genotype was done in 102 patients. All specimens were stained with hematoxylin-eosin, picrosirius and perls. Staging of hepatitis $\mathrm{C}$ was scored by Brazilian Classification and the diagnosis of non-alcoholic steatohepatitis by the American Association for the Study of Liver Diseases criteria. Results - Steatosis was found in 65 of 120 cases (54.2\%); it was mild in 37/65 (56.9\%), moderate in 12/65 (18.5\%) and severe in 10/65 (15.4\%). In relation to fibrosis, 80 of 120 patients had mild fibrosis F0-F2 (66.6\%) and 40 (33.3\%) had more advanced fibrosis (F3 or F4). Steatosis was associated with genotype $3(76.7 \%)$ in comparison with other genotypes $(49,0 \%)$. There were no relationship between steatosis and advanced fibrosis F3/F4 (52,5\%) or mild fibrosis (54,4\%). Non-alcoholic steatohepatitis was diagnosed in 8/120 cases (6.7\%) and was significantly associated with hepatitis $\mathrm{C}$ virus genotype 3 and with advanced fibrosis (F3 and F4). Conclusions - The presence of steatosis and non-alcoholic steatohepatitis in chronic hepatitis $\mathrm{C}$ are associated to genotype 3; moreover non-alcoholic steatohepatitis was correlated with more advanced fibrosis.

HEADINGS - Fatty liver. Hepatitis C, chronic. 


\section{REFERÊNCIAS BIBLIOGRÁFICAS}

1. Adinolfi LE, Gambardella M, Andreana A, Tripodi MF, Utili R, Ruggiero G. Steatosi accelerates the progression of liver damage of chronic hepatitis $\mathrm{C}$ patients and correlate with specific HCV genotype and visceral obesity. Hepatology. 2001;33:1358-64.

2. American Diabetes Association. Clinical practice recommendations 2000. Diabetes Care. 2000;23 (Suppl):1-8

3. Asselah T, Boyer N, Guimont M-C, Cazals-Hatem D, Tubach F, Nahon K, Daikha H, Vidaud D, Martinot M, Vidaud M, Degott C, Valla D, Marcellin P. Liver fibrosis is not associated with steatosis but with necroinflammation in French patients with chronic hepatitis C. Gut. 2003;52:1638-43.

4. Bach N, Thung SN, Schaffner. The histological features of chronic hepatitis C and autoimmune chronic hepatitis a comparative analysis. Hepatology. 1992;15:572-7.

5. Brunt EM, Janney C, Di Bisceglie A, Neuschwander-Tetri B, Bacon B. Nonalcoholic steatohepatitis: a proposal for grading and staging the histologic lesions. Am J Gastroenterol. 1999;94:2467-74.

6. Castéra L, Hézode C, Roudot-Thoraval F, Bastie A, Zafrani ES, Pawlotsky J, Dhumeaux $\mathrm{D}$. Worsening of steatosis is an independent factor of fibrosis progression in untreated patients with chronic hepatitis $\mathrm{C}$ and paired liver biopsies. Gut. 2003;52:288-92.

7. Castéra L, Hézode C, Roudot-Thoraval F, Lonjon I, Zafrani E-S, Pawlotsky J-M, Dhumeaux D. Effect of antiviral treatment on evolution of liver steatosis in patient with chronic hepatitis $\mathrm{C}$ : indirect evidence of a role of hepatitis $\mathrm{C}$ virus genotype 3 in steatosis. Gut. 2004;53:420-4.

8. Czaja AJ, Carpenter HA, Santrach PJ, Moore SB. Host- and disease-specific factor affecting steatosis in chronic hepatitis C. J Hepatol. 1998;29:198-206.

9. Diehl AM. Insulin resistence and steatosis in hepatitis $\mathrm{C}$ virus infection. Gut. 2005;54:903-6.

10. Fartoux L, Poujol-Robert A, Guechout J, Wendum D, Poupon R, Serfaty L. Insulin resistance is a cause of steatosis and fibrosis progression in chronic hepatitis C. Gut. 2005;54:1003-8

11. Friedenberg F, Pungpapong S, Zaeri N, Braitman L. The impact of diabetes and obesity on liver histology in patients with hepatitis C. Diabetes Obes Metab. 2003;5:150-5.

12. Gayotto LCC, Comitê SBP/SBH. Visão histórica e consenso nacional sobre a classificação das hepatites crônicas. Projeto do Clube de Patologia Hepática da Sociedade Brasileir de Patologia aprovado pela Sociedade Brasileira de Hepatologia. GED Gastroenterol Endosc Dig. 2000;19:137-40

13. Gayotto LCC. Esteato-hepatite não-alcoólica. In: Gayotto LCC, Alves VAF, editores Doenças do fígado e das vias biliares. São Paulo: Atheneu; 2001. cap.35.

14. Gayotto LCC, Alves VAF. Patologia das hepatites crônicas. In: Gayotto LCC, Alves VAF, editores. Doenças do fígado e das vias biliares. São Paulo: Atheneu, 2001. cap.49.

15. Gordom A, McLean C, Pedersen J, Bailey M, Roberts S. Hepatic steatosis in chronic hepatitis B and C: predictors, distribution and effect on fibrosis. J Hepatol. 2005;43:38-44.

16. Halfon P, Ouzan D, Cattan L, Cacoub P. The diagnostic tools of hepatitis C virus infections. Presse Med. 2004;33:538-41.

17. Hwang SJ, Luo J, Chu C, Lai C, Lu C, Tsay S, Wu J, Chang F, Lee S. Hepatic steatosis in chronic hepatitis $\mathrm{C}$ virus infection: prevalence and clinical correlation. J Gastroentero Hepatol. 2001;16;190-5.
18. Kumar D, Farrell G, Fung C, George J. Hepatitis C virus genotype 3 is cytopathic to hepatocytes reversal of hepatic steatosis after sustained therapeutic response. Hepatology. 2002;36:1266-72.

19. Lerat H, Honda M, Beard M, Loesch K, Sun J, Yang Y, Okuda M, Gosert R, Xiao $\mathrm{S}-\mathrm{Y}$, Weinman S, Lemon S. Steatosis and liver cancer in transgenic mice expressing the structural and nonstructural proteins of hepatitis $\mathrm{C}$ virus. Gastroenterology. 2002;122:352-65.

20. Liu CJ, Jeng YM, Chen PJ, Lai MY, Huang WL, Kao JH, Chen DS. Influence of metabolic syndrome, viral genotype and antiviral therapy on superimposed fatty liver disease in chronic hepatitis C. Antivir Ther. 2005;10:405-15.

21. Moriya K, Nakagawa K, Santa T, Shintani Y, Fujie H, Miyoshi H, Tsusumi T, Miyazawa $\mathrm{T}$, Ishibashi K, Horie T, Imai K, Todoroki T, Kimura S, Koike K. Oxidative stress in the absence of inflammation in a mouse model for hepatitis $\mathrm{C}$ virus-associated hepatocarcinogenesis. Cancer Res. 2001;61:4365-70.

22. Neuschwander-Tetri B, Caldwell S. Nonalcoholic steatohepatitis. Summary of an AASLD single topic conference. Hepatology. 2003;37:1202-19.

23. Okuda M, Li K, Beard M, Showalter L, Scholle F, Lemon S, Weinman SA. Mitochondrial injury, oxidative stress, and antioxidant gene expression are induced by hepatitis $\mathrm{C}$ virus core protein. Gastroenterology. 2002;122:366-75.

24. Poynard T, Ratziu V, McHutchison J, Manns M, Goodman Z, Zeuzem S, Younossi Z, Albrecht J. Effect of treatment with peginterferon or interferon alfa- $2 b$ and ribavirin on steatosis in patients infected with hepatitis C. Hepatology. 2003;38:75-85.

25. Rubbia-Brandt L, Fabris P, Paganin S, Leandro G, Male P-J, Giostra E, Carlotto A, Bozzola L, Smedile A, Negro F. Steatosis affects chronic hepatitis $\mathrm{C}$ progression in a genotype specific way. Gut. 2004;53:406-12.

26. Sanyal AJ. Non-alcoholic fatty liver disease and hepatitis C - risk factors and clinical implications. Aliment Pharmacol Ther. 2005;22 (suppl 2):48-51.

27. Scheuer PJ, Ashrafzadeh P, Sherlock S, Brown D, Duscheiko GM. The patology of hepatitis C. Hepatology. 1992;15:567-71.

28. Solis-Herruzo JA, Perez-Carreras M, Fernandez-Vazquez I, Garfia C, Bernardos E, Castellano G, Colina F. Factors associated with the presence of nonalcoholic steatohepatitis in patients with chronic hepatitis C. Am J Gastroenterol. 2005;100:1091-8.

29. Wanless IR, Lenz JS. Fatty liver hepatitis (steatohepatitis) and obesity an autopsy study with analysis of risk factors. Hepatology. 1990;12:1106-10.

30. Westin J, Nordlinder H, Lagging M, Norkrans G, Wejstal R. Steatosis accelerates fibrosis development over time in hepatitis $\mathrm{C}$ virus genotype 3 infected patients. J Hepatol. 2002;37:837-42

31. Wyatt J, Baker H, Prasad P, Gong YY, Millson C. Steatosis and fibrosis in patients with chronic hepatitis C. J Clin Pathol. 2004;57:402-6.

32. Younossi ZM, McCullough A, Ong JP, Barnes DS, Post A, Tavill A, Brigman D, Martin L, Assmann J, Gramlich T, Mullen K, O'Shea R, Cartey W, Ferguson R. Obesity and non-alcoholic fatty liver disease in chronic hepatitis C. J Clin Gastroenterol. 2004;38:705-9. 